\title{
FINITE BOUNDS FOR HÖLDER-BRASCAMP-LIEB MULTILINEAR INEQUALITIES
}

\author{
Jonathan Bennett, Anthony Carbery, Michael Christ, and Terence \\ TAO
}

AbStract. A criterion is established for the validity of multilinear inequalities of a class considered by Brascamp and Lieb, generalizing well-known inequalties of Rogers and Hölder, Young, and Loomis-Whitney.

\section{Formulation}

Consider multilinear functionals

$$
\Lambda\left(f_{1}, f_{2}, \cdots, f_{m}\right)=\int_{\mathbb{R}^{n}} \prod_{j=1}^{m} f_{j}\left(\ell_{j}(y)\right) d y
$$

where each $\ell_{j}: \mathbb{R}^{n} \rightarrow \mathbb{R}^{n_{j}}$ is a surjective linear transformation, and $f_{j}: \mathbb{R}^{n_{j}} \rightarrow$ $[0,+\infty]$. Let $p_{1}, \cdots, p_{m} \in[1, \infty]$. For which $m$-tuples of exponents and linear transformations is

$$
\sup _{f_{1}, \cdots, f_{m}} \frac{\Lambda\left(f_{1}, f_{2}, \cdots, f_{m}\right)}{\prod_{j}\left\|f_{j}\right\|_{L^{p_{j}}}}<\infty ?
$$

The supremum is taken over all $m$-tuples of nonnegative Lebesgue measurable functions $f_{j}$ having positive, finite norms. If $n_{j}=n$ for every index $j$ then (1.2) is essentially a restatement of Hölder's inequality. ${ }^{1}$ Other well-known particular cases include Young's inequality for convolutions and the Loomis-Whitney inequality ${ }^{2}[15]$.

In this paper we characterize finiteness of the supremum (1.2) in linear algebraic terms, and discuss certain variants and a generalization. The problem has a long history, including the early work of Rogers [17] and Hölder [12]. In this level of generality, the question was to our knowledge first posed by Brascamp and Lieb [4]. A primitive version of the problem involving Cartesian product rather than linear algebraic structure was posed and solved by Finner [10]; see $\S 7$ below. In the case when the dimension $n_{j}$ of each target space equals one, Barthe [1] characterized (1.2). Carlen, Lieb and Loss [7] gave an alternative characterization, closely related to ours, and an alternative proof for that case. [7] developed an inductive analysis

Received by the editors June, 3, 2005. Revision received November 3, 2008.

The third author was supported in part by NSF grant DMS-040126.

The fourth author is supported by a grant from the MacArthur Foundation, by NSF grant DMS0649473, and by the NSF Waterman award.

${ }^{1}$ For a discussion of the history of Hölder's inequality, including its discovery by Rogers [17], see [16].

${ }^{2}$ Loomis and Whitney considered only the special case where each $f_{j}$ is the characteristic function of a set. 
closely related to that of Finner, whose argument in turn relied on a slicing and induction argument employed earlier by Loomis and Whitney [15] and Calderón [6] to treat special cases. [7] also introduced a version of the key concepts of critical and subcritical subspaces, a higher-dimensional reformulation of which is essential in our work.

An alternative line of analysis exists. Although rearrangement inequalities such as that of Brascamp, Lieb, and Luttinger [5] do not apply when the target spaces have dimensions greater than one, Lieb [14] nonetheless showed that the supremum in (1.2) equals the supremum over all $m$-tuples of Gaussian functions, ${ }^{3}$ meaning those of the form $f_{j}=\exp \left(-Q_{j}(y, y)\right)$ for some positive definite quadratic form $Q_{j}$. See [7] and references cited there for more on this approach. In a companion paper [3] we have given other proofs of our characterization of (1.2), by using heat flow to continuously deform arbitrary functions $f_{j}$ to Gaussians while increasing the ratio in (1.2). That approach extends work of Carlen, Lieb, and Loss [7] via a method which they introduced.

We are indebted to a referee, whose careful reading and comments have improved the exposition.

\section{Results}

Denote by $\operatorname{dim}(V)$ the dimension of a vector space $V$, and by $\operatorname{codim}_{W}(V)$ the codimension of a subspace $V \subset W$ in $W$. It is convenient to reformulate the problem in a more invariant fashion. Let $H, H_{1}, \ldots, H_{m}$ be Hilbert spaces of finite, positive dimensions. Each is equipped with a canonical Lebesgue measure, by choosing orthonormal bases, thus obtaining identifications with $\mathbb{R}^{\operatorname{dim}(H)}, \mathbb{R}^{\operatorname{dim}\left(H_{j}\right)}$. Let $\ell_{j}: H \rightarrow H_{j}$ be surjective linear mappings. Let $f_{j}: H_{j} \rightarrow \mathbb{R}$ be nonnegative. Then $\Lambda\left(f_{1}, \cdots, f_{m}\right)$ equals $\int_{H} \prod_{j=1}^{m} f_{j} \circ \ell_{j}(y) d y$.

Theorem 2.1. For $1 \leq j \leq m$ let $H, H_{j}$ be Hilbert spaces of finite, positive dimensions. For each index $j$ let $\ell_{j}: H \rightarrow H_{j}$ be surjective linear transformations, and let $p_{j} \in[1, \infty]$. Then $(1.2)$ holds if and only if

$$
\operatorname{dim}(H)=\sum_{j} p_{j}^{-1} \operatorname{dim}\left(H_{j}\right)
$$

and

$$
\operatorname{dim}(V) \leq \sum_{j} p_{j}^{-1} \operatorname{dim}\left(\ell_{j}(V)\right) \text { for every subspace } V \subset H .
$$

This equivalence is established by other methods in [3], Theorem 1.15.

Given that (2.1) holds, the hypothesis (2.2) can be equivalently restated as

$$
\operatorname{codim}_{H}(V) \geq \sum_{j} p_{j}^{-1} \operatorname{codim}_{H_{j}}\left(\ell_{j}(V)\right) \text { for every subspace } V \subset H \text {; }
$$

\footnotetext{
${ }^{3}$ This situation should be contrasted with that of multilinear operators of the same general form, mapping $\otimes_{j} L^{p_{j}}$ to $L^{q}$. When $q \geq 1$, such multilinear operators are equivalent by duality to multilinear forms $\Lambda$. This is not so for $q<1$, and Gaussians are then quite far from being extremal [8].
} 
any two of these three conditions (2.1), (2.2), (2.3) imply the third. As will be seen through the discussion of variants below, (2.2) expresses a necessary condition governing large-scale geometry (compare Theorem 2.5), while (2.3) expresses a necessary condition governing small-scale geometry (compare Theorem 2.2). See also the discussion of necessary conditions for Theorem 2.3.

In the rank one case, when each target space $H_{j}$ is one-dimensional, a necessary and sufficient condition for inequality (1.2) was first obtained by Barthe [1]. Carlen, Lieb, and Loss [7] gave a different proof of the inequality for the rank one case, and a different characterization which is closely related to ours. Write $\ell_{j}(x)=\left\langle x, v_{j}\right\rangle$. It was shown in [7] that (1.2) is equivalent, in the rank one case, to having $\sum_{j} p_{j}^{-1}=\operatorname{dim}(H)$ and $\sum_{j \in S} p_{j}^{-1} \leq \operatorname{dim}\left(\operatorname{span}\left(\left\{v_{j}: j \in S\right\}\right)\right)$ for every subset $S$ of $\{1,2, \cdots, m\}$; a set of indices $S$ was said to be subcritical if this last inequality holds, and to be critical if it holds with equality. In the higher-rank case, we have formulated these concepts as properties of subspaces of $H$, rather than of subsets of $\{1,2, \cdots, m\}$.

To elucidate the connection between the two formulations in the rank one case, define $W_{S}=\operatorname{span}\left\{v_{j}: j \in S\right\}$, and say that a set of indices $S$ is maximal if there is no larger set $\tilde{S}$ of indices satisfying $W_{\tilde{S}}=W_{S}$. All sets of indices are subcritical, if and only if all maximal sets of indices are subcritical. If $j \in S$ then $\operatorname{codim}_{H_{j}}\left(\ell_{j}\left(W_{S}^{\perp}\right)\right)=1$; if $j \notin S$ and $S$ is maximal then $\operatorname{codim}_{H_{j}}\left(\ell_{j}\left(W_{S}^{\perp}\right)\right)=0$; and $\operatorname{codim}\left(W_{S}^{\perp}\right)=\operatorname{dim}\left(\operatorname{span}\left(\left\{v_{j}: j \in S\right\}\right)\right)$. Thus if $S$ is maximal, then the subcriticality of $S$ is equivalent to $\sum_{j=1}^{n} p_{j}^{-1} \operatorname{codim}_{H_{j}}\left(\ell_{j}\left(W_{S}^{\perp}\right)\right) \leq \operatorname{codim}\left(W_{S}^{\perp}\right)$. As noted above, under the condition $\sum_{j=1}^{n} p_{j}^{-1}=\operatorname{dim}(H)$, this is equivalent to our subcriticality condition $\operatorname{dim}(V) \leq \sum_{j} p_{j}^{-1} \operatorname{dim}\left(\ell_{j}(V)\right)$ for the subspace $V=W_{S}^{\perp}$.

The necessity of (2.1) follows from scaling: if $f_{j}^{\lambda}\left(x_{j}\right)=g_{j}\left(\lambda x_{j}\right)$ for each $\lambda \in$ $\mathbb{R}^{+}$then $\Lambda\left(\left\{f_{j}^{\lambda}\right\}\right)$ is proportional to $\lambda^{-\operatorname{dim}(H)}$, while $\prod_{j}\left\|f_{j}^{\lambda}\right\|_{p_{j}}$ is proportional to $\prod_{j} \lambda^{-\operatorname{dim}\left(H_{j}\right) / p_{j}}$. That $(2.2)$ is also necessary will be shown in $\S 5$ in the course of the proof of the more general Theorem 2.3.

Remark 2.1. $\Lambda$ can be alternatively expressed as a constant multiple of the integral $\int_{\Sigma} \prod_{j} f_{j} d \sigma$, where $\Sigma$ is a linear subspace of $\oplus_{j} H_{j}$ and $\sigma$ is Lebesgue measure on $\Sigma$. More exactly, $\Sigma$ is the range of the map $H \ni x \mapsto \oplus_{j} \ell_{j}(x)$. Denote by $\pi_{j}$ the restriction to $\Sigma$ of the natural projection $\pi_{j}: \oplus_{i} H_{i} \rightarrow H_{j}$. Then condition (2.2) can be restated as

$$
\operatorname{dim}(\tilde{\Sigma}) \leq \sum_{j} p_{j}^{-1} \operatorname{dim}\left(\pi_{j}(\tilde{\Sigma})\right) \text { for every linear subspace } \tilde{\Sigma} \subset \Sigma .
$$

A local variant is also natural. Consider

$$
\Lambda_{\text {loc }}\left(f_{1}, \cdots, f_{m}\right)=\int_{\{y \in H:|y| \leq 1\}} \prod_{j} f_{j} \circ \ell_{j}(y) d y .
$$

Theorem 2.2. Let $H, H_{j}, \ell_{j}$, and $f_{j}: H_{j} \rightarrow[0, \infty)$ be as in Theorem 2.1. Let $p_{j} \in[1, \infty]$ for $1 \leq j \leq m$. A necessary and sufficient condition for there to exist $C<\infty$ such that

$$
\Lambda_{\mathrm{loc}}\left(f_{1}, \cdots, f_{m}\right) \leq C \prod_{j}\|f\|_{L^{p_{j}}}
$$


for all nonnegative measurable functions $f_{j}$ is that every subspace $V$ of $H$ satisfies (2.3): $\operatorname{codim}_{H}(V) \geq \sum_{j} p_{j}^{-1} \operatorname{codim}_{H_{j}}\left(\ell_{j}(V)\right)$.

This is equivalent to Theorem 8.17 of [3], proved there by a different method.

Certain cases of Theorem 2.2 follow from Theorem 2.1; if there exist exponents $r_{j}$ satisfying the hypotheses (2.1) and (2.2) of Theorem 2.1, such that $r_{j} \leq p_{j}$ for all $j$, then the conclusion of Theorem 2.2 follows directly from that of Theorem 2.1 by Hölder's inequality, since $\left\|f_{j}\right\|_{L^{r_{j}}} \leq C^{\prime}\left\|f_{j}\right\|_{L^{p_{j}}}$. But not all cases of Theorem 2.2 are subsumed in Theorem 2.1 in this way. See Remark 7.1 for examples.

The next theorem, in which some but not necessarily all coordinates of $y$ are constrained to a bounded set, unifies Theorems 2.1 and 2.2.

Theorem 2.3. Let $H, H_{0}, \cdots, H_{m}$ be finite-dimensional Hilbert spaces and assume that $\operatorname{dim}\left(H_{j}\right)>0$ for all $j \geq 1$. Let $\ell_{j}: H \rightarrow H_{j}$ be linear transformations for $0 \leq j \leq m$, which are surjective for all $j \geq 1$. Let $p_{j} \in[1, \infty]$ for $1 \leq j \leq m$. Then there exists $C<\infty$ such that

$$
\int_{\left\{y \in H:\left|\ell_{0}(y)\right| \leq 1\right\}} \prod_{j=1}^{m} f_{j} \circ \ell_{j}(y) d y \leq C \prod_{j=1}^{m}\left\|f_{j}\right\|_{L^{p_{j}}}
$$

for all nonnegative Lebesgue measurable functions $f_{j}$ if and only if

$$
\operatorname{dim}(V) \leq \sum_{j=1}^{m} p_{j}^{-1} \operatorname{dim}\left(\ell_{j}(V)\right) \quad \text { for all subspaces } V \subset \operatorname{kernel}\left(\ell_{0}\right)
$$

and

$$
\operatorname{codim}_{H}(V) \geq \sum_{j=1}^{m} p_{j}^{-1} \operatorname{codim}_{H_{j}}\left(\ell_{j}(V)\right) \text { for all subspaces } V \subset H \text {. }
$$

This subsumes Theorem 2.2, by taking $H_{0}=H$ and $\ell_{0}: H \rightarrow H$ to be the identity; (2.8) then only applies to $\{0\}$, for which it holds automatically, so that the only hypothesis is then (2.9). On the other hand, Theorem 2.1 is the special case $\ell_{0} \equiv 0$ of Theorem 2.3. In that case kernel $\left(\ell_{0}\right)=H$, so (2.8) becomes (2.2). In addition, the case $V=\{0\}$ of (2.9) yields the reverse inequality $\operatorname{dim}(H) \geq \sum_{j} p_{j}^{-1} \operatorname{dim}\left(H_{j}\right)$. Thus the hypotheses of Theorem 2.3 imply those of Theorem 2.1 when $\ell_{0} \equiv 0$. The converse implication also holds, as was pointed out in the discussion of Theorem 2.2.

Our next result is one of several possible discrete analogues. Recall [13] that any finitely generated Abelian group $G$ is isomorphic to $\mathbb{Z}^{r} \times H$ for some integer $r$ and some finite Abelian group $H ; r$ is uniquely determined and is called the rank of $G$.

Theorem 2.4. Let $G$ and $\left\{G_{j}: 1 \leq j \leq m\right\}$ be finitely generated Abelian groups. Let $\varphi_{j}: G \rightarrow G_{j}$ be homomorphisms. Let $p_{j} \in[1, \infty]$. Then

$$
\operatorname{rank}(H) \leq \sum_{j} p_{j}^{-1} \operatorname{rank}\left(\varphi_{j}(H)\right) \text { for every subgroup } H \text { of } G
$$

if and only if there exists $C<\infty$ such that

$$
\sum_{y \in G} \prod_{j=1}^{m}\left(f_{j} \circ \varphi_{j}\right)(y) \leq C \prod_{j}\left\|f_{j}\right\|_{\ell^{p_{j}}\left(G_{j}\right)} \text { for all } f_{j}: G_{j} \rightarrow[0, \infty) .
$$


Here the $\ell^{p_{j}}$ norms are defined with respect to counting measure.

A special case arises when $G$ is isomorphic to $\mathbb{Z}^{d}, G_{j}$ is isomorphic to $\mathbb{Z}^{d_{j}}$ for all $j$, and each $\varphi_{j}$ is represented by a matrix with integer entries. The general case of Theorem 2.4 can be deduced directly from this special case, using the isomorphisms between e.g. $G$ and $\mathbb{Z}^{d} \times H$ for some finite group $H$, and the fact that all $\ell^{p}$ norms are mutually equivalent on finite sets.

A related variant is as follows. In $\mathbb{R}^{d}$, for each $n \in \mathbb{Z}^{d}$ define $Q_{n}=\left\{x \in \mathbb{R}^{d}\right.$ : $|x-n| \leq \sqrt{d}\}$. The space $\ell^{p}\left(L^{\infty}\right)\left(\mathbb{R}^{d}\right)$ is the space of all $f \in L^{\infty}\left(\mathbb{R}^{d}\right)$ for which $\left(\sum_{n \in \mathbb{Z}^{d}}\|f\|_{L^{\infty}\left(Q_{n}\right)}^{p}\right)^{1 / p}$ is finite.

Theorem 2.5. Let $m \geq 1$ be a positive integer, and for each $j \in\{1,2, \cdots, m\}$ let $\ell_{j}: \mathbb{R}^{d} \rightarrow \mathbb{R}^{d_{j}}$ be a surjective linear transformation. Let $p_{j} \in[1, \infty]$. Then

$$
\operatorname{dim}(V) \leq \sum_{j} p_{j}^{-1} \operatorname{dim}\left(\ell_{j}(V)\right) \text { for every subspace } V \subset \mathbb{R}^{d}
$$

if and only if there exists $C<\infty$ such that

$$
\int_{\mathbb{R}^{d}} \prod_{j=1}^{m}\left(f_{j} \circ \ell_{j}\right)(y) d y \leq C \prod_{j}\left\|f_{j}\right\|_{\ell^{p_{j}}\left(L^{\infty}\right)\left(\mathbb{R}^{d_{j}}\right)}
$$

for all measurable $f_{j}: \mathbb{R}^{d_{j}} \rightarrow[0, \infty)$.

A related result is Corollary 8.11 of [3].

Yet another variant of our results, based on Cartesian product rather than linear algebraic or group theoretic structure, has been obtained earlier by Finner [10]; see also [11] for a discussion of some special cases from another point of view. Let $\left\{\left(X_{i}, \mu_{i}\right)_{i \in I}\right\}$ be a finite collection of measure spaces, and let $(X, \mu)=\prod_{i \in I}\left(X_{i}, \mu_{i}\right)$ be their product. Let $J$ be another finite index set. For each $j \in J$, let $S_{j}$ be some nonempty subset of $I$. Let $Y_{j}=\prod_{i \in S_{j}} X_{i}$, equipped with the associated product measure, and let $\pi_{j}: X \rightarrow Y_{j}$ be the natural projection map. Let $f_{j}: Y_{j} \rightarrow[0, \infty]$ be measurable. To avoid trivialities, we assume throughout the discussion that $I, J$ are nonempty and that $\mu(X)$ is strictly positive. Define

$$
\Lambda\left(f_{j}\right)_{j \in J}=\int_{X} \prod_{j \in J} f_{j} \circ \pi_{j} d \mu .
$$

Denote by $|\cdot|$ the cardinality of a finite set.

Let $p_{j} \in[1, \infty]$ for each $j \in J$. Finner's theorem then asserts that if

$$
1=\sum_{j: i \in S_{j}} p_{j}^{-1} \text { for all } i \in I
$$

then

$$
\Lambda\left(f_{j}\right)_{j \in J} \leq \prod_{j \in J}\left\|f_{j}\right\|_{L^{p_{j}}\left(Y_{j}\right)} .
$$

A modest generalization of Finner's theorem is discussed in $\S 7$.

The hypothesis (2.15) can be equivalently restated as

$$
|K|=\sum_{j \in J} p_{j}^{-1}\left|S_{j} \cap K\right| \text { for every subset } K \subset I,
$$


or again as the conjunction of $|I|=\sum_{j \in J} p_{j}^{-1}\left|S_{j}\right|$ and $|K| \leq \sum_{j \in J} p_{j}^{-1}\left|S_{j} \cap K\right|$ for every $K \subset I$. When each space $X_{i}$ is some Euclidean space equipped with Lebesgue measure, the hypotheses in this last form are precisely those of Theorem 2.1, specialized to this limited class of linear mappings. The analogue of a subspace is now a subset $K \subset I$, and the analogue of criticality is (2.17); thus (2.16) holds if and only if every subset $K$ is critical. This contrasts with the situations treated by Barthe [1], by Carlen, Lieb, and Loss [7], and in Theorem 2.1, where generic subspaces will be subcritical even if critical subspaces exist.

A special case treated by Calderón [6] is as follows: Let $1 \leq k<n$. Let $x=$ $\left(x_{1}, \cdots, x_{n}\right)$ be coordinates for $\mathbb{R}^{n}$. For each subset $S \subset\{1,2,3, \cdots, n\}$ of cardinality $k$ let $\mathbb{R}_{S}^{k}$ be a copy of $\mathbb{R}^{k}$, with coordinates $\left(x_{i}\right)_{i \in S}$. Let $\pi_{S}: \mathbb{R}^{n} \rightarrow \mathbb{R}^{k}$ be the natural projections. Then for arbitrary nonnegative measurable functions,

$$
\int_{\mathbb{R}^{n}} \prod_{S} f_{S}\left(\pi_{S}(x)\right) d x \leq \prod_{S}\left\|f_{S}\right\|_{L^{p}\left(\mathbb{R}_{S}^{k}\right)}
$$

where $p=\left(\begin{array}{l}n-1 \\ k-1\end{array}\right)$. A particular instance of Calderón's theorem is the Loomis-Whitney inequality

$$
\int_{\mathbb{R}^{n}} \prod_{j=1}^{n} f_{j} \circ \pi_{j}(x) d x \leq \prod_{j=1}^{n}\left\|f_{j}\right\|_{L^{n-1}},
$$

where $\pi_{j}: \mathbb{R}^{n} \rightarrow \mathbb{R}^{n-1}$ is the mapping that forgets the $j$-th coordinate.

Two quite distinct investigations motivated our interest in these problems. One derives from work [2] of three of us on multilinear versions of the Kakeya-Nikodym maximal functions. A second motivator was work [9] on multilinear operators with additional oscillatory factors; see Proposition 3.1 and Corollary 3.2 below.

\section{An application to oscillatory integrals}

Proposition 3.1. Let $m>1$. For $1 \leq j \leq m$ let $\ell_{j}: \mathbb{R}^{n} \rightarrow \mathbb{R}^{n_{j}}$ be surjective linear mappings. Let $P: \mathbb{R}^{n} \rightarrow \mathbb{R}$ be a polynomial. Let $\varphi \in C_{0}^{1}\left(\mathbb{R}^{n}\right)$ be a compactly supported, continuously differentiable cutoff function. For $\lambda \in \mathbb{R}$ and $f_{j} \in L^{p_{j}}\left(\mathbb{R}^{n_{j}}\right)$ define $\Lambda_{\lambda}\left(f_{1}, \cdots, f_{m}\right)=\int_{\mathbb{R}^{n}} e^{i \lambda P(x)} \prod_{j=1}^{m} f_{j}\left(\ell_{j}(x)\right) \varphi(x) d x$. Suppose that there exist $\delta>0$ and $C<\infty$ such that for all functions $f_{j} \in L^{\infty}$ and all $\lambda \in \mathbb{R}$

$$
\left|\Lambda_{\lambda}\left(f_{1}, \cdots, f_{m}\right)\right| \leq C|\lambda|^{-\delta} \prod_{j=1}^{m}\left\|f_{j}\right\|_{L^{\infty}} .
$$

Let $\left(p_{1}, \cdots, p_{m}\right) \in[1, \infty]^{m}$, and suppose that for every proper subspace $V \subset \mathbb{R}^{n}$,

$$
\operatorname{codim}_{\mathbb{R}^{n}}(V)>\sum_{j} p_{j}^{-1} \operatorname{codim}_{\mathbb{R}^{n_{j}}}\left(\ell_{j}(V)\right) .
$$

Then there exist $\delta>0$ and $C<\infty$, depending on $\left(p_{1}, \cdots, p_{m}\right)$, such that

$$
\left|\Lambda_{\lambda}\left(f_{1}, \cdots, f_{m}\right)\right| \leq C|\lambda|^{-\delta} \prod_{j=1}^{m}\left\|f_{j}\right\|_{L^{p_{j}}}
$$

for all parameters $\lambda \in \mathbb{R}$ and functions $f_{j} \in L^{p_{j}}\left(\mathbb{R}^{n_{j}}\right)$. 
By Theorem 2.2, the condition that $\operatorname{codim}_{\mathbb{R}^{n}}(V) \geq \sum_{j} p_{j}^{-1} \operatorname{codim}_{\mathbb{R}^{n}}\left(\ell_{j}(V)\right)$ for every subspace $V \subset \mathbb{R}^{n}$ guarantees that the integral defining $\Lambda_{\lambda}\left(f_{1}, \cdots, f_{m}\right)$ converges absolutely for all functions $f_{j} \in L^{p_{j}}$, and is bounded by $C \prod_{j}\left\|f_{j}\right\|_{L^{p_{j}}}$. The conclusion of Proposition 3.1 then follows directly from this inequality and the hypothesis by complex interpolation.

A polynomial $P$ is said [9] to be nondegenerate, relative to the collection $\left\{\ell_{j}\right\}$ of mappings, if $P$ cannot be expressed as $P=\sum_{j} P_{j} \circ \ell_{j}$ for any collection of polynomials $P_{j}: \mathbb{R}^{n_{j}} \rightarrow \mathbb{R}$.

Corollary 3.2. Let $\left\{\ell_{j}\right\}, P, \varphi$ be as in Proposition 3.1. Suppose that $P$ is nondegenerate relative to $\left\{\ell_{j}\right\}$. Suppose that either $(i) n_{j}=1$ for all $j, m<2 n$, and the family $\left\{\ell_{j}\right\}$ of mappings is in general position, or (ii) $n_{j}=n-1$ for all $j$. Let $\left(p_{1}, \cdots, p_{m}\right) \in[1, \infty]^{m}$ and suppose that for every proper subspace $V \subset \mathbb{R}^{n}$, $\operatorname{codim}_{\mathbb{R}^{n}}(V)>\sum_{j} p_{j}^{-1} \operatorname{codim}_{\mathbb{R}^{n_{j}}}\left(\ell_{j}(V)\right)$. Then there exists $\delta>0$ such that for any $\varphi \in C_{0}^{1}$ there exists $C<\infty$ such that for all functions $f_{j} \in L^{p_{j}}\left(\mathbb{R}^{n_{j}}\right)$,

$$
\left|\Lambda_{\lambda}\left(f_{1}, \cdots, f_{m}\right)\right| \leq C|\lambda|^{-\delta} \prod_{j=1}^{m}\left\|f_{j}\right\|_{L^{p_{j}}} .
$$

Here general position means that for any subset $S \subset\{1,2, \cdots, m\}$ of cardinality $|S| \leq n, \cap_{j \in S}$ kernel $\left(\ell_{j}\right)$ has dimension $n-|S|$.

By Theorems 2.1 and 2.2 of [9], the hypotheses imply (3.1). Proposition 3.1 then implies the Corollary.

\section{Proof of sufficiency in Theorem 2.1}

We begin with the proof of sufficiency of the hypotheses $(2.1),(2.2)$ for the finiteness of the supremum in (1.2). Necessity will be established in the next section.

The next definition is made for the purposes of the discussion of Theorem 2.1; alternative notions of criticality are appropriate for the other theorems.

Definition 4.1. Relative to a set of exponents $\left\{p_{j}\right\}$, a subspace $V \subset H$ is said to be critical if

$$
\operatorname{dim}(V)=\sum_{j} p_{j}^{-1} \operatorname{dim}\left(\ell_{j}(V)\right),
$$

to be supercritical if the right-hand side is less than $\operatorname{dim}(V)$, and to be subcritical if the right-hand side is greater than $\operatorname{dim}(V)$.

In this language, the hypothesis (2.1) states that $V=H$ is critical relative to $\left\{p_{j}\right\}$, while (2.2) states that no subspace of $H$ is supercritical.

Proof of sufficiency in Theorem 2.1. The proof proceeds by induction on the dimension of $H$. When $\operatorname{dim}(H)=1$, necessarily $\operatorname{dim}\left(H_{j}\right)=1$ for all $j$. The hypothesis of the theorem in this case is that $\sum_{j} p_{j}^{-1}=1$, and the conclusion is simply a restatement of Hölder's inequality for functions in $L^{p_{j}}\left(\mathbb{R}^{1}\right)$.

Suppose now that $\operatorname{dim}(H)>1$. There are two cases. Case 1 arises when there exists some proper nonzero critical subspace $W \subset H$. The analysis then relies on a factorization procedure visible in the work of Calderón [6], Finner [10], and Carlen, 
Lieb, and Loss [7]. Express $H=W^{\perp} \oplus W$ where $W^{\perp}$ is the orthocomplement of $W$, with coordinates $y=\left(y^{\prime}, y^{\prime \prime}\right) \in W^{\perp} \oplus W$; we will identify $\left(y^{\prime}, 0\right)$ with $y^{\prime}$ and $\left(0, y^{\prime \prime}\right)$ with $y^{\prime \prime}$. Define $U_{j} \subset H_{j}$ to be

$$
U_{j}=\ell_{j}(W) .
$$

Define $\tilde{\ell}_{j}=\left.\ell_{j}\right|_{W}: W \rightarrow U_{j}$, which is surjective. For $y^{\prime} \in W^{\perp}$ and $x_{j} \in U_{j}$ define

$$
g_{j, y^{\prime}}\left(x_{j}\right)=f_{j}\left(x_{j}+\ell_{j}\left(y^{\prime}\right)\right) .
$$

Then

$$
f_{j}\left(\ell_{j}\left(y^{\prime}, y^{\prime \prime}\right)\right)=f_{j}\left(\ell_{j}\left(y^{\prime}\right)+\tilde{\ell}_{j}\left(y^{\prime \prime}\right)\right)=g_{j, y^{\prime}}\left(\tilde{\ell}_{j}\left(y^{\prime \prime}\right)\right) .
$$

Now

$$
\begin{aligned}
\Lambda\left(f_{1}, \cdots, f_{m}\right) & =\int_{W^{\perp}} \int_{W} \prod_{j} f_{j}\left(\ell_{j}\left(y^{\prime}, y^{\prime \prime}\right)\right) d y^{\prime \prime} d y^{\prime} \\
& =\int_{W^{\perp}} \int_{W} \prod_{j} g_{j, y^{\prime}}\left(\tilde{\ell}_{j}\left(y^{\prime \prime}\right)\right) d y^{\prime \prime} d y^{\prime}
\end{aligned}
$$

so

$$
\Lambda\left(f_{1}, \cdots, f_{m}\right)=\int_{W^{\perp}} \tilde{\Lambda}\left(g_{1, y^{\prime}}, \cdots, g_{m, y^{\prime}}\right) d y^{\prime}
$$

where

$$
\tilde{\Lambda}\left(g_{1}, \cdots, g_{m}\right)=\int_{W} \prod_{j} g_{j}\left(\tilde{\ell}_{j}\left(y^{\prime \prime}\right)\right) d y^{\prime \prime}
$$

We claim that

$$
\tilde{\Lambda}\left(g_{1}, \cdots, g_{m}\right) \leq C \prod_{j}\left\|g_{j}\right\|_{p_{j}} .
$$

Since $W$ has dimension strictly less than $\operatorname{dim}(H)$, this follows from the induction hypothesis provided that $W$ is critical and no subspace $V \subset W$ is supercritical, relative to the mappings $\tilde{\ell}_{j}$ and exponents $p_{j}$. But since $\tilde{\ell}_{j}$ is the restriction of $\ell_{j}$ to $W$, this condition is simply the specialization of the original hypothesis from arbitrary subspaces of $H$ to those subspaces contained in $W$, together with the criticality of $W$ hypothesized in Case 1. Thus

$$
\Lambda\left(f_{1}, \cdots, f_{m}\right)=\int_{W^{\perp}} \tilde{\Lambda}\left(g_{1, y^{\prime}}, \cdots, g_{m, y^{\prime}}\right) d y^{\prime} \leq C \int_{W^{\perp}} \prod_{j}\left\|g_{j, y^{\prime}}\right\|_{L^{p_{j}}\left(U_{j}\right)} d y^{\prime}
$$

We will next show how this last integral is another instance of the original problem, with $H$ replaced by the lower-dimensional vector space $W^{\perp}$. For $z_{j} \in U_{j}^{\perp}$ define

$$
F_{j}\left(z_{j}\right)=\left(\int_{U_{j}} f_{j}\left(x_{j}+z_{j}\right)^{p_{j}} d x_{j}\right)^{1 / p_{j}}
$$

recalling that $f_{j} \geq 0$, with $F_{j}\left(z_{j}\right)=\operatorname{ess} \sup _{x_{j} \in U_{j}} f_{j}\left(x_{j}+z_{j}\right)$ if $p_{j}=\infty$. Thus ${ }^{4}$

$$
\left\|F_{j}\right\|_{L^{p_{j}}\left(U_{j}^{\perp}\right)}=\left\|f_{j}\right\|_{L^{p_{j}}\left(H_{j}\right)} .
$$

\footnotetext{
${ }^{4}$ If $U_{j}=\{0\}$ then the domain of $F_{j}$ is $H_{j}$, and $F_{j} \equiv f_{j}$. If $U_{j}=H_{j}$ then the domain of $F_{j}$ is $\{0\}$, and $\left\|F_{j}\right\|_{p_{j}}$ is by definition $F_{j}(0)=\left\|f_{j}\right\|_{p_{j}}$.
} 
Denote by $\pi_{U_{j}^{\perp}}: H_{j} \rightarrow U_{j}^{\perp}$ and $\pi_{U_{j}}: H_{j} \rightarrow U_{j}$ the orthogonal projections. Define $L_{j}: W^{\perp} \rightarrow U_{j}^{\perp}$ by

$$
L_{j}=\pi_{U_{j}^{\perp}} \circ \ell_{j} .
$$

Decomposing $\ell_{j}\left(y^{\prime}\right)=L_{j}\left(y^{\prime}\right)+u_{j}$ where $u_{j}=\pi_{U_{j}}\left(\ell_{j}\left(y^{\prime}\right)\right)$, and making the change of variables $\tilde{x}_{j}=x_{j}+u_{j}$ in $U_{j}$, gives (if $p_{j}<\infty$ )

$$
\begin{aligned}
& \quad\left\|g_{j, y^{\prime}}\right\|_{L^{p_{j}}\left(U_{j}\right)}^{p_{j}}=\int_{U_{j}}\left|g_{j, y^{\prime}}\left(x_{j}\right)\right|^{p_{j}} d x_{j}=\int_{U_{j}}\left|f_{j}\left(x_{j}+\ell_{j}\left(y^{\prime}\right)\right)\right|^{p_{j}} d x_{j} \\
& =\int_{U_{j}}\left|f_{j}\left(x_{j}+u_{j}+L_{j}\left(y^{\prime}\right)\right)\right|^{p_{j}} d x_{j}=\int_{U_{j}}\left|f_{j}\left(\tilde{x}_{j}+L_{j}\left(y^{\prime}\right)\right)\right|^{p_{j}} d \tilde{x}_{j}=F_{j}\left(L_{j}\left(y^{\prime}\right)\right)^{p_{j}} .
\end{aligned}
$$

Consequently we have shown thus far that

$$
\Lambda\left(f_{1}, \cdots, f_{m}\right) \leq C \int_{W^{\perp}} \prod_{j} F_{j} \circ L_{j}
$$

where $\left\|F_{j}\right\|_{L^{p_{j}\left(U_{j}^{\perp}\right)}}=\left\|f_{j}\right\|_{L^{p_{j}\left(H_{j}\right)}}$. Since $\ell_{j}: H \rightarrow H_{j}$ is surjective, $H_{j}$ is spanned by $\ell_{j}(W)=U_{j}$ together with $\ell_{j}\left(W^{\perp}\right)$; thus the orthogonal projection of $\ell_{j}\left(W^{\perp}\right)$ onto $U_{j}^{\perp}$ is all of $U_{j}^{\perp}$; thus each $L_{j}: W^{\perp} \rightarrow U_{j}^{\perp}$ is surjective.

To complete the argument for Case 1 we need only show that

$$
\int_{W^{\perp}} \prod_{j} F_{j} \circ L_{j} \leq C \prod_{j}\left\|F_{j}\right\|_{L^{p_{j}}\left(U_{j}^{\perp}\right)} .
$$

By induction on the ambient dimension, this follows from the next lemma, which appears in [7] in the special case when $\operatorname{dim}\left(H_{j}\right)=1$ for all $j$. Although there are no additional complications in the general case, we include a proof for the sake of completeness.

Lemma 4.1. Fix an m-tuple $\left(p_{1}, \cdots, p_{m}\right)$ of exponents in $[1, \infty]$. Suppose that with respect to these exponents, $H$ is critical with respect to these exponents, $H$ has no supercritical subspaces, and $W \subset H$ is a nonzero proper critical subspace. Define surjective linear transformations $L_{j}=\pi_{\ell_{j}(W)^{\perp}} \circ \ell_{j}: W^{\perp} \rightarrow \ell_{j}(W)^{\perp}$. Then for any subspace $V \subset W^{\perp}, \operatorname{dim}(V) \leq \sum_{j} p_{j}^{-1} \operatorname{dim}\left(L_{j}(V)\right)$.

Proof. Let $V$ be any subspace of $H$ contained in $W^{\perp}$. Associate to $V$ the subspace $V+W \subset H$. Since $V \subset W^{\perp}, \operatorname{dim}(V+W)=\operatorname{dim}(V)+\operatorname{dim}(W)$. Moreover, for any $j$,

$$
\operatorname{dim}\left(\ell_{j}(V+W)\right)=\operatorname{dim}\left(L_{j}(V)\right)+\operatorname{dim}\left(\ell_{j}(W)\right),
$$

since $L_{j}=\pi_{\ell_{j}(W)^{\perp}} \circ \ell_{j}$.

Therefore

$$
\begin{aligned}
\sum_{j} p_{j}^{-1} \operatorname{dim}\left(L_{j}(V)\right) & =\sum_{j} p_{j}^{-1} \operatorname{dim}\left(\ell_{j}(V+W)\right)-\sum_{j} p_{j}^{-1} \operatorname{dim}\left(\ell_{j}(W)\right) \\
& =\sum_{j} p_{j}^{-1} \operatorname{dim}\left(\ell_{j}(V+W)\right)-\operatorname{dim}(W) \\
& \geq \operatorname{dim}(V+W)-\operatorname{dim}(W)=\operatorname{dim}(V),
\end{aligned}
$$


by the criticality of $W$ and subcriticality of $V+W$. Thus $V$ is not supercritical.

When $V=W^{\perp}$, one has $V+W=H$, whence $\sum_{j} p_{j}^{-1} \operatorname{dim}\left(\ell_{j}(V+W)\right)=\operatorname{dim}(V+$ $W$ ) since $H$ is assumed to be critical. With this information the final inequality of the preceding display becomes an equality, demonstrating that $W^{\perp}$ is critical.

The proof of Case 1 of Theorem 2.1 is complete. Turn next to Case 2, in which every nonzero proper subspace of $H$ is subcritical. $\infty^{-1}$ is to be interpreted as zero throughout the discussion.

Consider the set $K$ of all $m$-tuples $t=\left(t_{1}, \cdots, t_{m}\right) \in[0,1]^{m}$ such that relative to the exponents $p_{j}=t_{j}^{-1}, H$ is critical and has no supercritical subspace. Thus $K$ equals the intersection of $[0,1]^{m}$ with the hyperplane defined by the equation $\operatorname{dim}(H)=\sum_{j} t_{j} \operatorname{dim}\left(H_{j}\right)$, and with all of the closed half-spaces defined by the inequalities $\operatorname{dim}(V) \leq \sum_{j} t_{j} \operatorname{dim}\left(\ell_{j}(V)\right)$, as $V$ ranges over all subspaces of $H$. Therefore $K$ is comvex and compact.

While the number of such subspaces $V$ is infinite, the number of $m+1$-tuples $\left(\operatorname{dim}(V), \operatorname{dim}\left(\ell_{1}(V)\right), \cdots, \operatorname{dim}\left(\ell_{m}(V)\right)\right)$ is finite. The set of all distinct inequalities induced by subspaces of $H$ is in one-to-one corresondence with the set of all such $m+1$-tuples. Thus $K$ is the intersection of $[0,1]^{m}$ with a hyperplane and with finitely many closed half-spaces. Therefore $K$ has finitely many extreme points. Since $K$ is compact and convex, $K$ consequently equals the convex hull of its extreme points.

We will show that for any extreme point $t$ of $K$, there exists a finite constant $C$ such that $\Lambda\left(f_{1}, \cdots, f_{m}\right) \leq C \prod_{j}\left\|f_{j}\right\|_{L^{1 / t_{j}}}$ for all nonnegative measurable functions $f_{j}$. Granting such inequalities, let $\left\{t^{(i)}\right\}_{i}$ be the set of all extreme points of $K$, and let $C_{i}$ be constants for which the corresponding inequalities hold. Any $t=\left(t_{1}, \cdots, t_{m}\right) \in K$ can be expressed as $t=\sum_{i} \lambda_{i} t^{(i)}$ for some scalars $\lambda_{i} \in[0,1]$ satisfying $\sum_{i} \lambda_{i}=1$. Write $t^{(i)}=\left(t_{1}^{(i)}, \cdots, t_{m}^{(i)}\right)$. A direct application of complex interpolation shows that

$$
\Lambda\left(f_{1}, \cdots, f_{m}\right) \leq \prod_{i} C_{i}^{\lambda_{i}} \prod_{j}\left\|f_{j}\right\|_{L^{1 / t_{j}}}^{\lambda_{i}}=C \prod_{j}\left\|f_{j}\right\|_{L^{1 / t_{j}}}
$$

for all nonnegative measurable functions $f_{j}$, where $C=\prod_{i} C_{i}^{\lambda_{i}}$.

At an arbitrary extreme point $t$ of $K$, at least one of the inequalities defining $K$ must become an equality. Therefore some nonzero proper subspace of $H$ must be critical relative to $t$, or $t_{i} \in\{0,1\}$ for at least one index $i$.

Consider the set $\tilde{K}$ of all $t \in[0, \infty)^{m}$ for which $\operatorname{dim}(H)=\sum_{j} t_{j} \operatorname{dim}\left(H_{j}\right)$ and $\operatorname{dim}(V) \leq \sum_{j} t_{j} \operatorname{dim}\left(\ell_{j}(V)\right)$ for all subspaces $V$ of $H$. Thus $K=\tilde{K} \cap[0,1]^{m} \subset \tilde{K}$. We claim that $\tilde{K}=K$. Indeed, for any $t \in \tilde{K}$, the homogeneity and subcriticality conditions imply that $\operatorname{codim}_{H}(V) \geq \sum_{j} t_{j} \operatorname{codim}_{H_{j}}\left(\ell_{j}(V)\right)$ for all subspaces $V \subset H$. Consider any index $i$ and let $V$ be the nullspace of $\ell_{i}$. Then

$$
\begin{aligned}
\operatorname{dim}\left(H_{i}\right)=\operatorname{codim}_{H}(V) \geq \sum_{j} t_{j} \operatorname{codim}_{H_{j}}\left(\ell_{j}(V)\right) & \\
& \geq t_{i} \operatorname{codim}_{H_{i}}\{0\}=t_{i} \operatorname{dim}\left(H_{i}\right) .
\end{aligned}
$$

Therefore $t_{i} \leq 1$ for all $i$, so $t \in K$. Thus $\tilde{K} \subset K$, as claimed.

Since $\tilde{K}=K$, if $t$ is any extreme point of $K$, then either equality must hold in at least one of the inequalities defining $\tilde{K}$, or the number $m$ of indices $j$ must be 1 
(so that the hypothesis $\operatorname{dim}(H)=\sum_{j} p_{j}^{-1} \operatorname{dim}\left(H_{j}\right)$ specifies the single exponent $p_{1}^{-1}$, reducing $K$ to a single point). Thus some nonzero proper subspace of $H$ must be critical relative to $t$, or at least one coordinate $t_{i}$ must equal 0 , or $m=1$. In the first subcase we are in Case 1, which has already been treated above. For the case $m=1$, see the second paragraph below.

In the second subcase, we may proceed by induction on $m$, for an inequality $\Lambda\left(f_{1}, \cdots, f_{m}\right) \leq C\left\|f_{i}\right\|_{L^{\infty}} \prod_{j \neq i}\left\|f_{j}\right\|_{L^{p_{j}}}$ is equivalent to

$$
\Lambda\left(f_{1}, \cdots, f_{i-1}, 1, f_{i+1}, \cdots, f_{m}\right) \leq C \prod_{j \neq i}\left\|f_{j}\right\|_{L^{p_{j}}} .
$$

The hypotheses of Theorem 2.1 are inherited by this multilinear operator of one lower degree, acting on $\left\{f_{j}: j \neq i\right\}$, whence the desired inequality follows by induction.

This induction is founded by the subcase in which $m=1$. In this case the hypothe$\operatorname{sis} \operatorname{dim}(H)=\sum_{j} p_{j}^{-1} \operatorname{dim}\left(H_{j}\right)$ becomes $\operatorname{dim}(H)=p_{1}^{-1} \operatorname{dim}\left(H_{1}\right)$. Since $\ell_{1}: H \rightarrow H_{1}$ is assumed to be surjective, $\operatorname{dim}\left(H_{1}\right) \leq \operatorname{dim}(H)$, so this forces both $p_{1}=1$ and $\operatorname{dim}\left(H_{1}\right)=\operatorname{dim}(H)$. Since $\ell_{1}: H \rightarrow H_{1}$ is surjective, it must be invertible. Therefore $\Lambda\left(f_{1}\right)=\int_{H} f_{1} \circ \ell_{1}=c \int_{H_{1}} f_{1}=c\left\|f_{1}\right\|_{L^{1}}$ for some finite constant $c$, which certainly implies the desired inequality $\Lambda\left(f_{1}\right) \leq C\left\|f_{1}\right\|_{L^{1}}$.

Remark 4.1. When $\operatorname{dim}\left(H_{j}\right)=1$ for all $j$, every extreme point $\left(p_{1}^{-1}, \cdots, p_{m}^{-1}\right)$ of $K$ has each $p_{j}^{-1} \in\{0,1\}[1],[7]$. This is not the case in general; in the Loomis-Whitney inequality (2.19) for $\mathbb{R}^{n}, K$ consists of a single point, with $p_{j}=n-1$ for all $j$.

\section{Proof of Theorem 2.3}

Consider $\int_{\left\{y \in H:\left|\ell_{0}(y)\right| \leq 1\right\}} \prod_{j=1}^{m} f_{j} \circ \ell_{j} d y$ where the linear transformation $\ell_{0}$ has domain $H$ and range $H_{0}$, with $\operatorname{dim}\left(H_{0}\right)$ possibly equal to zero. Thus some components of $y$ are constrained to a bounded set, while the rest are free. Set

$$
\mathcal{V}=\operatorname{kernel}\left(\ell_{0}\right)
$$

the component of $y$ lying in $\mathcal{V}$ is completely unconstrained, while the component in $\mathcal{V}^{\perp}$ is constrained to a bounded set.

Proof of necessity of (2.8) and (2.9). For any subspace $V \subset H$ define $V_{\text {big }}=V \cap \mathcal{V}$ and $V_{\text {small }}=V \ominus V_{\text {big }}=V \cap\left(V_{\text {big }}\right)^{\perp}$, so that $V=V_{\text {small }} \oplus V_{\text {big. }}$. Denote by $\pi_{V}$ the orthogonal projection of either $H$ or some $H_{j}$ onto a subspace $V$.

Let $r \leq 1 \leq R$ be arbitrary. Define $f_{j}=f_{j}\left(x_{j}\right)$ to be the characteristic function of the set $S_{j}$ of all $x_{j} \in H_{j}$ such that

$$
\left|\pi_{\ell_{j}\left(V_{\text {big }}\right)}\left(x_{j}\right)\right| \leq R,\left|\pi_{\ell_{j}(V) \cap\left(\ell_{j}\left(V_{\text {big }}\right)\right)^{\perp}}\left(x_{j}\right)\right| \leq 1 \text {, and }\left|\pi_{\left(\ell_{j}(V)\right)^{\perp}}\left(x_{j}\right)\right| \leq r \text {. }
$$

Let $c_{0}>0$ be a small constant independent of $r, R$, and define $S \subset H$ to be the set of all $y$ such that $\left|\pi_{V^{\perp}}(y)\right| \leq c_{0} r,\left|\pi_{V_{\text {small }}}(y)\right| \leq c_{0}$, and $\left|\pi_{V_{\text {big }}}(y)\right| \leq c_{0} R$.

Fix a constant $C<\infty$ such that $\left|\ell_{j}(y)\right| \leq C|y|$ for all $y, j$. Provided that $c_{0}<1 / 3 C$, $y \in S \Rightarrow f_{j}\left(\ell_{j}(y)\right)=1$ for all indices $j$. Indeed, for any $y \in S \cap V^{\perp},\left|\ell_{j}(y)\right| \leq$ $C|y| \leq C c_{0} r<r / 3$, so $\ell_{j}(y) \in \frac{1}{3} S_{j}$. If on the other hand $y \in S \cap V_{\text {small }}$, then $\left|\ell_{j}(y)\right| \leq C|y| \leq C c_{0}<\frac{1}{3}$, so since $\ell_{j}(y) \in \ell_{j}(V), \ell_{j}(y) \in \frac{1}{3} S_{j}$. Finally if $y \in S \cap V_{\text {big }}$ then $\left|\ell_{j}(y)\right| \leq C c_{0} R$, which implies that $\ell_{j}(y) \in \frac{1}{3} S_{j}$ since $\ell_{j}(y) \in \ell_{j}\left(V_{\text {big }}\right)$. Any $y \in S$ admits an orthogonal decomposition $y=u+v+w$ where $u \in S \cap V^{\perp}, v \in S \cap V_{\text {small }}$, 
and $w \in S \cap V_{\text {big. }}$. These components satisfy $|u| \leq c_{0} r,|v| \leq c_{0}$, and $|w| \leq c_{0} R$, by definition of $S$. $\ell_{j}(y)$ is thus a sum of three terms in $\frac{1}{3} S_{j}$, so $\ell_{j}(y) \in S_{j}$.

Moreover $y \in S \Rightarrow\left|\ell_{0}(y)\right| \leq 1$. Therefore

$$
\tilde{\Lambda}_{\text {loc }}\left(\left\{f_{j}\right\}\right) \geq|S| \sim R^{\operatorname{dim}\left(V_{\text {big }}\right)} \cdot r^{\operatorname{codim}_{H}(V)}
$$

while

$$
\left\|f_{j}\right\|_{p_{j}} \sim R^{p_{j}^{-1} \operatorname{dim}\left(\ell_{j}\left(V_{\mathrm{big}}\right)\right)} r^{p_{j}^{-1} \operatorname{codim}_{H_{j}}\left(\ell_{j}(V)\right)} .
$$

Suppose that the ratio $\tilde{\Lambda}_{\text {loc }}\left(\left\{f_{j}\right\}\right) / \prod_{j}\left\|f_{j}\right\|_{p_{j}}$ is bounded uniformly as a function of $r, R$. By letting $R \rightarrow \infty$ while $r$ remains fixed, we conclude that

$$
\operatorname{dim}\left(V_{\mathrm{big}}\right) \leq \sum_{j} p_{j}^{-1} \operatorname{dim}\left(\ell_{j}\left(V_{\mathrm{big}}\right)\right) .
$$

Letting $r \rightarrow 0$ with $R$ fixed gives

$$
\operatorname{codim}_{H}(V) \geq \sum_{j} p_{j}^{-1} \operatorname{codim}_{H_{j}}\left(\ell_{j}(V)\right) .
$$

The following lemma will be used in the proof of Theorem 2.3.

Lemma 5.1. Suppose that $\operatorname{codim}_{H}(V) \geq \sum_{j} p_{j}^{-1} \operatorname{codim}_{H_{j}}\left(\ell_{j}(V)\right)$ for every subspace $V \subset H$, and that $W \subset H$ is a subspace satisfying $\operatorname{codim}_{H}(W)=$ $\sum_{j} p_{j}^{-1} \operatorname{codim}_{H_{j}}\left(\ell_{j}(W)\right)$. Then for any subspace $V \subset W$,

$$
\operatorname{codim}_{W}(V) \geq \sum_{j} p_{j}^{-1} \operatorname{codim}_{\ell_{j}(W)}\left(\ell_{j}(V)\right) .
$$

Likewise for any subspace $V \subset W^{\perp}$,

$$
\operatorname{codim}_{W^{\perp}}(V) \geq \sum_{j} p_{j}^{-1} \operatorname{codim}_{\ell_{j}(W)^{\perp}}\left(L_{j}(V)\right) .
$$

Proof. For the first conclusion,

$$
\begin{aligned}
\operatorname{codim}_{W}(V) & =\operatorname{dim}(W)-\operatorname{dim}(V)=\operatorname{codim}_{H}(V)-\operatorname{codim}_{H}(W) \\
& \geq \sum_{j} p_{j}^{-1} \operatorname{codim}_{H_{j}}\left(\ell_{j}(V)\right)-\sum_{j} p_{j}^{-1} \operatorname{codim}_{H_{j}}\left(\ell_{j}(W)\right) \\
= & \sum_{j} p_{j}^{-1}\left(\operatorname{dim}\left(\ell_{j}(W)\right)-\operatorname{dim}\left(\ell_{j}(V)\right)\right)=\sum_{j} p_{j}^{-1} \operatorname{codim}_{\ell_{j}(W)}\left(\ell_{j}(V)\right) .
\end{aligned}
$$


For the second conclusion,

$$
\begin{aligned}
\operatorname{codim}_{W^{\perp}}(V) & =\operatorname{dim}(H)-\operatorname{dim}(W)-\operatorname{dim}(V) \\
& =\operatorname{codim}_{H}(V+W) \\
& \geq \sum_{j} p_{j}^{-1} \operatorname{codim}_{H_{j}}(V+W) \\
& =\sum_{j} p_{j}^{-1}\left(\operatorname{dim}\left(H_{j}\right)-\operatorname{dim}\left(\ell_{j}(W)\right)-\operatorname{dim}\left(L_{j}(V)\right)\right) \\
& =\sum_{j} p_{j}^{-1}\left(\operatorname{dim}\left(L_{j}\left(W^{\perp}\right)\right)-\operatorname{dim}\left(L_{j}(V)\right)\right) . \\
& =\sum_{j} p_{j}^{-1} \operatorname{codim}_{L_{j}\left(W^{\perp}\right)}\left(L_{j}(V)\right) .
\end{aligned}
$$

The identity $\operatorname{dim}\left(H_{j}\right)=\operatorname{dim}\left(\ell_{j}(W)\right)+\operatorname{dim}\left(L_{j}\left(W^{\perp}\right)\right)$ used to obtain the final line is (4.15) specialized to $V=W^{\perp}$.

Proof of sufficiency in Theorem 2.3. The proof follows the inductive scheme of the proof of Theorem 2.1. To simplify notation set $t_{j}=p_{j}^{-1} \in[0,1]$. Case 1 now breaks down into two subcases. Case 1A arises when there exists a nonzero proper subspace $W$ of $H$ that is contained in $\mathcal{V}$ and is critical in the sense of (2.8), that is, ${ }^{5}$ $\sum_{j} t_{j} \operatorname{dim}\left(\ell_{j}(W)\right)=\operatorname{dim}(W)$.

With coordinates $\left(y^{\prime}, y^{\prime \prime}\right)$ for $W^{\perp} \oplus W, \ell_{0}$ is independent of $y^{\prime \prime}$, and for every subspace $V \subset W, \sum_{j} t_{j} \operatorname{dim}\left(\ell_{j}(V)\right) \geq \operatorname{dim}(V)$ by (2.8). Thus the collection of mappings $\left\{\left.\ell_{j}\right|_{W}\right\}$ satisfies the hypothesis of Theorem 2.1, whence $\int_{W} \prod_{j} f_{j} \circ \ell_{j}\left(y^{\prime}, y^{\prime \prime}\right) d y^{\prime \prime} \leq$ $C \prod_{j} F_{j}\left(y^{\prime}\right)$ where $\left\|F_{j}\right\|_{L^{p_{j}}\left(W^{\perp}\right)} \leq C\left\|f_{j}\right\|_{L^{p_{j}}\left(H_{j}\right)}$.

It remains to bound $\int_{W^{\perp}} \chi_{B} \circ \ell_{0}\left(y^{\prime}, 0\right) \prod_{j} F_{j} \circ L_{j}\left(y^{\prime}\right) d y^{\prime}$, where $B$ denotes the characteristic function of a ball of finite radius. Theorem 2.3 can be invoked by induction on the ambient dimension, provided that (2.8) and (2.9) hold for the data $W^{\perp}, \mathcal{V} \cap W^{\perp},\left\{U_{j}^{\perp}, L_{j}, p_{j}\right\}$. We will write $(2.8)_{H},(2.8)_{W}$, and $(2.8)_{W^{\perp}}$ to distinguish between this hypothesis for the three different data that arise in the discussion; likewise for (2.9).

$(2.9)_{W}$ is the condition that $\operatorname{codim}_{W^{\perp}}(V) \geq \sum_{j} t_{j} \operatorname{codim}_{L_{j}\left(W^{\perp}\right)}\left(L_{j}(V)\right)$ for every subspace $V \subset W^{\perp}$, which is the second conclusion of Lemma 5.1. (2.8) $W$ is the condition

$$
\operatorname{dim}(V) \leq \sum_{j} t_{j} \operatorname{dim}\left(L_{j}(V)\right) \text { for all subspaces } V \subset \mathcal{V} \cap W^{\perp} .
$$

Since $V, W$ are both contained in $\mathcal{V}$ so is $V+W$, so $\sum_{j} t_{j} \operatorname{dim}\left(\ell_{j}(V+W)\right) \geq \operatorname{dim}(V+$ $W)=\operatorname{dim}(V)+\operatorname{dim}(W)$ by $(2.8)_{H}$. This together with the previously established identity $\operatorname{dim}\left(\ell_{j}(V+W)\right)=\operatorname{dim}\left(\ell_{j}(W)\right)+\operatorname{dim}\left(L_{j}(V)\right)$ and the criticality condition $\sum_{j} t_{j} \operatorname{dim}\left(\ell_{j}(W)\right)=\operatorname{dim}(W)$ yields (5.6). Thus Case $1 \mathrm{~A}$ is treated by applying Theorem 2.1 for $W$ and the induction hypothesis for $W^{\perp}$.

Case $1 \mathrm{~B}$ arises when there exists a nonzero proper subspace $W \subset H$ that is critical in the sense of $(2.9)$, that is, $\operatorname{codim}_{H}(W)=\sum_{j} t_{j} \operatorname{codim}_{H_{j}}\left(\ell_{j}(W)\right)$. The analysis

\footnotetext{
${ }^{5}$ All summations with respect to $j$ are taken over $1 \leq j \leq m$.
} 
follows the same inductive scheme. Lemma 5.1 guarantees that $(2.9)_{W}$ holds, while $(2.8)_{W}$ is simply the specialization of $(2.8)_{H}$ to subspaces $V \subset W \cap \mathcal{V}$. Thus Theorem 2.3 may be applied by induction to $W,\left\{\ell_{j}(W)\right\},\left\{\left.\ell_{j}\right|_{W}\right\},\left\{p_{j}\right\}$.

This reduces matters to $\int_{W^{\perp} \cap\left\{\left|L_{0}\left(y^{\prime}\right)\right| \leq 1\right\}} \prod_{j} F_{j} \circ L_{j} d y^{\prime}$, where the nullspace $\tilde{V}$ of $L_{0}$ is the set of all $y^{\prime} \in W^{\perp}$ for which there exists $y^{\prime \prime} \in W$ such that $\ell_{0}\left(y^{\prime}, y^{\prime \prime}\right)=0$; thus the subspace $\mathcal{V} \subset H$ is now replaced by $\pi_{W^{\perp}} \mathcal{V} \subset W^{\perp}$.

Now it is natural to expect to use $(2.8)_{H}$ to establish $(2.8)_{W^{\perp}}$, but the latter pertains to certain subspaces not contained in $\mathcal{V}$, about which the former says nothing. Luckily the inequality in (5.6) holds for arbitrary subspaces $V \subset W^{\perp}$, not merely those contained in $\pi_{W} \mathcal{V}$. Indeed,

$$
\begin{aligned}
\sum_{j} t_{j} \operatorname{dim}\left(L_{j}(V)\right) & =\sum_{j} t_{j} \operatorname{dim}\left(\ell_{j}(V+W)\right)-\sum_{j} t_{j} \operatorname{dim}\left(\ell_{j}(W)\right) \\
& =\sum_{j} t_{j} \operatorname{codim}_{H_{j}}\left(\ell_{j}(W)\right)-\sum_{j} t_{j} \operatorname{codim}_{H_{j}}\left(\ell_{j}(V+W)\right) \\
& =\operatorname{codim}_{H}(W)-\sum_{j} t_{j} \operatorname{codim}_{H_{j}}\left(\ell_{j}(V+W)\right) \\
& \geq \operatorname{codim}_{H}(W)-\operatorname{codim}_{H}(V+W) \\
& =\operatorname{dim}(V) .
\end{aligned}
$$

The assumption that $W$ is critical in the sense that equality holds in $(2.9)_{H}$ implies (2.9) $)_{W}^{\perp}$, by the second conclusion of Lemma 5.1. Thus by induction on the dimension, Theorem 2.3 may be applied to the integral over $W^{\perp}$, concluding the proof for Case $1 \mathrm{~B}$.

Case 2 arises when no subspace $W$ is critical in either sense. Consider the set $K \subset[0,1]^{m}$ of all $\left(t_{1}, \cdots, t_{m}\right)$ such that $\sum_{j} t_{j} \operatorname{dim}\left(\ell_{j}(V)\right) \geq \operatorname{dim}(V)$ for all subspaces $V \subset \mathcal{V}=\operatorname{kernel}\left(\ell_{0}\right)$, and $\operatorname{codim}_{H}(V) \geq \sum_{j} t_{j} \operatorname{codim}_{H_{j}}\left(\ell_{j}(V)\right)$ for all subspaces $V \subset H$. As in the proof of Theorem 2.1, $K$ is a compact convex set with finitely many extreme points, and consequently equals the convex hull of the set of all of those extreme points. It suffices to prove that $\int_{H} \chi_{B} \circ \ell_{0} \prod_{j \geq 1} f_{j} \circ \ell_{j} \leq C \prod_{j}\left\|f_{j}\right\|_{q_{j}}$ for every extreme point $\left(t_{1}, \cdots, t_{m}\right)$ of $K$, where $q_{j}=t_{j}^{-1}$. Consider such an extreme point. If there exists a nonzero proper subspace $V \subset \mathcal{V}$ that is critical in the sense that $\sum_{j} t_{j} \operatorname{dim}\left(\ell_{j}(V)\right)=\operatorname{dim}(V)$, or a nonzero proper subspace $V \subset H$ that is critical in the sense that $\operatorname{codim}_{H}(V)=\sum_{j} t_{j} \operatorname{codim}_{H_{j}}\left(\ell_{j}(V)\right)$, then Case 1A or Case 1B apply.

There are other cases in which equality might hold in (2.8) or (2.9), besides those subsumed under Case 1. If equality holds for $V=\{0\}$ in (2.9) with $p_{j}^{-1}=t_{j}$, then $\operatorname{dim}(H)=\sum_{j} t_{j} \operatorname{dim}\left(H_{j}\right)$, which is the first hypothesis of Theorem 2.1. In conjunction with (2.9) this implies that (2.8) holds for every subspace $V \subset H$, which is the second hypothesis of Theorem 2.1. Therefore the conclusion (2.7) of Theorem 2.3 holds without the restriction $\left|\ell_{0}(y)\right| \leq 1$ in the integral, by Theorem 2.1.

If on the other hand $H=\mathcal{V}=\operatorname{kernel}\left(\ell_{0}\right)$ and equality holds for $V=H$ in (2.8) with $p_{j}^{-1}=t_{j}$, then $\operatorname{dim}(H)=\sum_{j} t_{j} \operatorname{dim}\left(H_{j}\right)$, so Theorem 2.1 applies once more.

Therefore matters reduce to the case where equality holds in (2.8) for no subspace of $\mathcal{V}$ except $V=\{0\}$, and where furthermore equality holds in (2.9) for no subspace 
of $H$ except for $V=H$ itself. Equality always holds in both of those cases, so they play no part in defining $K$.

$t$ satisfies $\left.\operatorname{codim}_{H}(V) \geq \sum_{j} t_{j} \operatorname{codim}_{H_{j}}(V)\right)$ for every subspace $V \subset H$. Therefore as in Case 2 of the proof of Theorem 2.1, every remaining extreme point $\left(t_{1}, \cdots, t_{m}\right)$ of $K$ must have $t_{i}=0$ for at least one index $i$.

By induction on $m$, it therefore suffices to treat the case $m=1$, with $p_{1}=\infty$. By (2.8) applied to $V=\operatorname{kernel}\left(\ell_{0}\right), \operatorname{dim}\left(\operatorname{kernel}\left(\ell_{0}\right)\right) \leq 0 \operatorname{dim}\left(H_{1}\right)=0$, so $\ell_{1}$ has no kernel. Therefore the restriction $\left|\ell_{0}(y)\right| \leq 1$ constrains $y$ to a bounded region, whence $\int_{\left|\ell_{0}(y)\right| \leq 1} f_{1} \circ \ell_{1}(y) d y \leq C\left\|f_{1}\right\|_{L^{\infty}}$ for some finite constant $C$.

\section{Proof of Theorem 2.4}

This proof contains no significant new elements. We denote the identity element of a group by $0 . \times$ will denote the Abelian direct product, that is, the Cartesian product of two Abelian groups, equipped with the Abelian group structure associated naturally to its factors. A subgroup $H$ of $G$ is said to be of finite index if the quotient group $G / H$ is finite. If $H, H^{\prime}$ are subgroups of $G$, then $H+H^{\prime}$ denotes the subgroup of $G$ generated by $H \cup H^{\prime}$.

A very few properties of finitely generated Abelian groups will be used in the proof. See for instance [13] pp. 76-80, especially Theorem 2.6. Let $G$ be any finitely generated Abelian group. $G$ is isomorphic to $\mathbb{Z}^{r} \times H$ for some integer $r$ and some finite Abelian group $H$ for a unique nonnegative integer $r$, called the rank of $G$. $H$ is uniquely determined up to isomorphism, and is isomorphic to the subgroup of $G$ consisting of all elements of finite order, which is called the torsion subgroup of $G$. Thus $G$ is finite if and only if $\operatorname{rank}(G)=0$. Any subgroup $H$ of $G$ is finitely generated, and satisfies $\operatorname{rank}(H) \leq \operatorname{rank}(G)$. If $H_{1}, H_{2}$ are subgroups of $G$, and if $H_{1} \cap H_{2}=\{0\}$, then $\operatorname{rank}\left(H_{1}+H_{2}\right)=\operatorname{rank}\left(H_{1}\right)+\operatorname{rank}\left(H_{2}\right)$. For any homomorphism $\varphi, \operatorname{rank}(\varphi(G)) \leq$ $\operatorname{rank}(G)$. Any subgroup $H$ is of course normal, so $G / H$ is also a finitely generated Abelian group; however, in contrast to the theory of vector spaces, $G$ is not in general isomorphic to the direct product of $H \times(G / H)$. However, the following weaker property does hold, and will serve as a substitute in our analysis: If $\pi: G \rightarrow G / H$ denotes the natural projection then for any subgroup $H^{\prime}$ of $G / H, \operatorname{rank}\left(\pi^{-1}\left(H^{\prime}\right)\right)=$ $\operatorname{rank}(H)+\operatorname{rank}\left(H^{\prime}\right)$. In particular, $\operatorname{rank}(G)=\operatorname{rank}(H)+\operatorname{rank}(G / H)$.

Let groups $G, G_{j}$, homomorphisms $\varphi_{j}$, and exponents $p_{j}$ satisfy the hypotheses of Theorem 2.4. Consider first the case where there exists a subgroup $G^{\prime} \subset G$, satisfying $0<\operatorname{rank}\left(G^{\prime}\right)<\operatorname{rank}(G)$, which is critical in the sense that $\sum_{j} p_{j}^{-1} \operatorname{rank}\left(\varphi_{j}\left(G^{\prime}\right)\right)=$ $\operatorname{rank}\left(G^{\prime}\right)$. Define $G_{j}^{\prime}=\varphi_{j}\left(G^{\prime}\right) \subset G_{j}$. Since every subgroup of $G$ inherits the hypothesis of the theorem, we may conclude by induction on the rank that

$$
\sum_{y \in G^{\prime}} \prod_{j}\left(f_{j} \circ \varphi_{j}\right)(y) \leq C \prod_{j}\left\|f_{j}\right\|_{\ell^{p_{j}}\left(G_{j}^{\prime}\right)} .
$$

Define $F_{j} \in \ell^{p_{j}}\left(G_{j} / G_{j}^{\prime}\right)$ by

$$
F_{j}\left(x+G_{j}^{\prime}\right)=\left(\sum_{z \in G_{j}^{\prime}}\left|f_{j}(x+z)\right|^{p_{j}}\right)^{1 / p_{j}} .
$$


Then $\left\|F_{j}\right\|_{\ell^{p_{j}}\left(G_{j} / G_{j}^{\prime}\right)}=\left\|f_{j}\right\|_{\ell^{p_{j}}\left(G_{j}\right)}$. Define homomorphisms $\psi_{j}: G / G^{\prime} \rightarrow G_{j} / G_{j}^{\prime}$ by composing each $\varphi_{j}$ with the quotient map from $G_{j}$ to $G_{j}^{\prime}$. Then

$$
\begin{aligned}
\sum_{y \in G} \prod_{j}\left(f_{j} \circ \varphi_{j}\right)(y) & =\sum_{x \in G / G^{\prime}} \sum_{z \in G^{\prime}} \prod_{j}\left(f_{j} \circ \varphi_{j}\right)(x+z) \\
& \leq C \sum_{x \in G / G^{\prime}} \prod_{j}\left(F_{j} \circ \psi_{j}\right)(x) ;
\end{aligned}
$$

the inequality follows from an invocation of (6.1). It suffices to show that the homomorphisms $\psi_{j}$ inherit the hypothesis of Theorem 2.4, which may then be applied by induction on the rank to yield the desired bound $O\left(\prod_{j}\left\|F_{j}\right\|_{\ell^{p_{j}}}\right)$. This hypothesis is verified using the criticality of $G^{\prime}$ and the additivity of ranks, just as additivity of dimensions was used in the proof of Theorem 2.1. Thus Theorem 2.4 is proved in the special case where there exists a critical subgroup $G^{\prime} \subset G$ satisfying $0<\operatorname{rank}\left(G^{\prime}\right)<\operatorname{rank}(G)$.

In the general case of Theorem 2.4, consider the compact convex set $K$ of all $\left(t_{1}, \cdots, t_{m}\right) \in[0,1]^{m}$ for which $\operatorname{rank}(H) \leq \sum_{j} t_{j} \operatorname{rank}\left(\varphi_{j}(H)\right)$ for all subgroups $H \subset$ $G$. As in the proof of Theorem 2.1, the set of all extreme points of $K$ is finite, and $K$ is equal to its convex hull. ${ }^{6}$ It suffices to prove that $\sum_{y \in G} \prod_{j}\left(f_{j} \circ \varphi_{j}\right)(y) \leq C \prod_{j}\left\|f_{j}\right\|_{1 / t_{j}}$ for all extreme points $\left(t_{1}, \cdots, t_{m}\right)$ of $K$.

If $\left(t_{1}, \cdots, t_{m}\right)$ is an extreme point then either $\sum_{j} t_{j} \operatorname{rank}\left(\varphi_{j}\left(G^{\prime}\right)\right)=\operatorname{rank}\left(G^{\prime}\right)$ for some subgroup $G^{\prime}$ satisfying $0<\operatorname{rank}\left(G^{\prime}\right)<\operatorname{rank}(G)$, or $t_{j} \in\{0,1\}$ for all indices $j$, or the total number $m$ of indices $j$ equals one. The first case has already been treated above.

Suppose that $\left(t_{1}, \cdots, t_{m}\right) \in K$ and $t_{j} \in\{0,1\}$ for all $j$. Let $S=\left\{j: t_{j}=1\right\}$, and consider the subgroup $G^{\prime}=\cap_{j: t_{j}=1} \operatorname{kernel}\left(\varphi_{j}\right)$. The hypothesis $(2.10)$ states that $0=\sum_{j \in S} \operatorname{rank}\left(\varphi_{j}\left(G^{\prime}\right)\right) \geq \operatorname{rank}\left(G^{\prime}\right)$, so $G^{\prime}$ has rank 0 , hence is finite. For any point $z=\left(z_{j}\right)_{j \in S} \in \prod_{j \in S} G_{j}$, the cardinality of $\left\{y \in G: \phi_{j}(y)=z_{j} \forall j \in S\right\}$ is $\leq\left|G^{\prime}\right|$. Therefore

$$
\begin{aligned}
\sum_{y \in G} \prod_{j}\left(f_{j} \circ \varphi_{j}\right)(y) & \leq \prod_{j \notin S}\left\|f_{j}\right\|_{\ell^{\infty}} \sum_{y \in G} \prod_{j \in S}\left(f_{j} \circ \varphi_{j}\right)(y) \\
& =\prod_{j \notin S}\left\|f_{j}\right\|_{\ell^{\infty}} \sum_{z \in \prod_{j \in S} G_{j}}\left|\left\{y: \varphi_{j}(y)=z_{j} \forall j \in S\right\}\right| \prod_{j \in S} f_{j}\left(z_{j}\right) \\
& \leq\left|G^{\prime}\right| \prod_{j \notin S}\left\|f_{j}\right\|_{\ell^{\infty}} \prod_{j \in S}\left\|f_{j}\right\|_{\ell^{1}}
\end{aligned}
$$

which is the desired inequality.

If $m=1$ then the hypothesis $\operatorname{rank}(G)=p_{1}^{-1} \operatorname{rank}\left(\varphi_{1}(G)\right)$ forces $p_{1}=1$ and $\operatorname{rank}\left(\varphi_{1}(G)\right)=\operatorname{rank}(G)$. Therefore the kernel of $\varphi_{1}$ has rank 0 , that is, it is a finite group. The required inequality $\sum_{y \in G}\left(f_{1} \circ \varphi_{1}\right)(y) \leq C\left\|f_{1}\right\|_{\ell^{1}}$ is then immediate.

\footnotetext{
${ }^{6}$ There is a subtlety here. The set of all subspaces of fixed dimension of a finite-dimensional vector space naturally carries the structure of a compact topological space. The set of all subgroups of a finitely generated Abelian group lacks such structure. However, the inequalities which define $K$ here are in one-to-one corresondence with the set of all $m$-tuples $\left(\operatorname{rank}(H), \operatorname{rank}\left(\varphi_{1}(H)\right), \cdots, \operatorname{rank}\left(\varphi_{m}(H)\right)\right)$, as $H$ varies over all subgroups of $G$. Since all ranks belong to the finite set $[0, \operatorname{rank}(G)] \cap \mathbb{Z}$, only finitely many such inequalities arise.
} 
Conversely, necessity of the hypothesis that $\operatorname{rank}(H) \leq \sum_{j} p_{j}^{-1} \operatorname{rank}\left(\varphi_{j}(H)\right)$ for all subgroups $H$ of $G$ is routine. Observe first that if the conclusion of Theorem 2.4 holds, then it holds with $G$ replaced by any subgroup $H$; this follows simply by restricting the sum over all $y \in G$ on the left-hand side of (2.11) to $y \in H$. Therefore in order to prove necessity of the hypothesis, it suffices to prove that $\operatorname{rank}(G) \leq \sum_{j} p_{j}^{-1} \operatorname{rank}\left(\varphi_{j}(G)\right)$.

It is no loss of generality to replace $G_{j}$ by $\varphi_{j}(G)$, so we may assume that each homomorphism $\varphi_{j}$ is surjective. Each group $G_{j}$ is isomorphic to $\mathbb{Z}^{r_{j}} \times T_{j}$ where $r_{j}=\operatorname{rank}\left(G_{j}\right)$, and $T_{j}$ is some finite group. Write $\left(x_{j}, t_{j}\right)$ for coordinates on $\mathbb{Z}^{r_{j}} \times T_{j}$, and define $\left\|\left(x_{j}, t_{j}\right)\right\|=\left|x_{j}\right|$. Define a similar function $G \ni y \mapsto\|y\|$. Let $R$ be an arbitrary large positive real number, and set $f_{j}\left(x_{j}, t_{j}\right)=1$ if $\left|x_{j}\right| \leq R$, and $=0$ otherwise. Then $\left\|f_{j}\right\|_{p_{j}} \sim R^{r_{j} / p_{j}}$ for large $R$. On the other hand, there exists $c>0$ independent of $R$ such that $\prod_{j}\left(f_{j} \circ \varphi_{j}\right)(y)=1$ for all $y \in G$ satisfying $\|y\| \leq c R$. The number of such points $y \in G$ is $\geq c^{\prime} R^{\operatorname{rank}(G)}$. By letting $R \rightarrow \infty$ we conclude that if (2.11) holds, then $\operatorname{rank}(G) \leq \sum_{j} p_{j}^{-1} \operatorname{rank}\left(G_{j}\right)$.

Outline of proof of Theorem 2.5. This argument requires no essentially new ideas. Necessity of the hypothesis for a subspace $V$ is proved by defining each $f_{j}$ to be the intersection of $\left\{x_{j}:\left|x_{j}\right| \leq R\right\}$ with $\left\{x_{j}\right.$ : distance $\left.\left(x_{j}, \ell_{j}(V)\right) \leq C_{0}\right\}$, and letting $R \rightarrow \infty$ while $C_{0}$ remains fixed.

The proof of sufficiency is based on an inductive argument for the critical case, in which there exists a proper subspace of positive dimension satisfying $\operatorname{dim}(V)=$ $\sum_{j} p_{j}^{-1} \operatorname{dim}\left(\ell_{j}(V)\right)$, and a direct verification for the case in which all $p_{j} \in\{0,1\}$. The latter is straightforward.

The former is more awkward than the corresponding step in our other proofs, because the intersection of $\mathbb{Z}^{d}$ with a subspace of $\mathbb{R}^{d}$ is rarely a cocompact lattice. Let $V$ be a nonzero critical subspace of $\mathbb{R}^{d}$. For each index $j$, define $W_{j}$ to be the orthocomplement in $\mathbb{R}^{d_{j}}$ of $\ell_{j}(V)$. Choose a sublattice $\mathcal{L}_{j}$ of $W_{j}$ of $\operatorname{rank} \operatorname{dim}\left(W_{j}\right)$, and a sublattice $\mathcal{L}_{j}^{\prime}$ of $\ell_{j}(V)$ of rank equal to $\operatorname{dim}\left(\ell_{j}(V)\right)$. Let $A, A^{\prime}<\infty$ be large constants. For $y \in W_{j}$ define

$$
F_{j}(y)=\left(\sum_{n \in \mathcal{L}^{\prime}} \sup _{|y-n| \leq A}\left|f_{j}(y+n)\right|^{p_{j}}\right)^{1 / p_{j}} .
$$

Define $\left\|F_{j}\right\|_{\ell^{p_{j}}\left(L^{\infty}\right)}$ by first taking the $L^{\infty}$ norm over $\left\{y:|y-n|<A^{\prime}\right\}$ for each $n \in \mathcal{L}$, then taking an $\ell^{p_{j}}$ norm with respect to $n$. Such a norm, associated to any sublattice of full rank, is comparable to such a norm associated to any other sublattice of full rank, provided that the constants $A^{\prime}$ are chosen to be sufficiently large, depending on the sublattices in question. For any $A, A^{\prime}<\infty$ there exists $C<\infty$ such that

$$
\left\|F_{j}\right\|_{\ell^{p_{j}}\left(L^{\infty}\right)\left(\ell_{j}(V)\right)} \leq C\left\|f_{j}\right\|_{\ell^{p_{j}}\left(L^{\infty}\right)\left(\mathbb{R}^{d_{j}}\right)} .
$$

If $A$ is sufficiently large then uniformly for all $y \in V^{\perp}$,

$$
\int_{V} \prod_{j}\left(f_{j} \circ \ell_{j}\right)(y+z) d z \leq C_{A^{\prime}} \prod_{j}\left(F_{j} \circ L_{j}\right)(y)
$$


where $L_{j}: V^{\perp} \rightarrow W_{j}$ is $\left.\ell_{j}\right|_{V^{\perp}}$ followed by orthogonal projection onto $W_{j}$. Thus

$$
\Lambda\left(f_{1}, \cdots, f_{m}\right) \leq C_{A} \tilde{\Lambda}\left(F_{1}, \cdots, F_{m}\right)=\int_{V^{\perp}} \prod_{j}\left(F_{j} \circ L_{j}\right) .
$$

If the constant $A^{\prime}$ appearing in the definition of the $\ell^{p_{j}}\left(L^{\infty}\right)$ norms of the functions $F_{j}$ is chosen to be sufficiently large, then there exists $C<\infty$ such that

$$
\tilde{\Lambda}\left(F_{1}, \cdots, F_{m}\right) \leq C \prod_{j}\left\|F_{j}\right\|_{\ell^{p_{j}}\left(L^{\infty}\right)} .
$$

The rest of the argument is as in our other proofs.

\section{Variants based on product structure}

Our next result is analogous to a unification of Theorems 2.3 and 2.5. We say that a measure space $(X, \mu)$ is atomic if there exists $\delta>0$ such that $\mu(E) \geq \delta$ for every measurable set $E$ having strictly positive measure.

Proposition 7.1. Suppose that the index set $I$ is a disjoint union $I=I_{0} \cup I_{\infty} \cup I_{\star}$, where $X_{i}$ is a finite measure space for each $i \in I_{0}$, is atomic for each $i \in I_{\infty}$, and is an arbitrary measure space for each $i \in I_{\star}$. Then a sufficient condition for the inequality (2.16) is that

$$
\begin{aligned}
& 1 \geq \sum_{j: i \in S_{j}} p_{j}^{-1} \text { for all } i \in I_{0} \\
& 1 \leq \sum_{j: i \in S_{j}} p_{j}^{-1} \text { for all } i \in I_{\infty} \\
& 1=\sum_{j: i \in S_{j}} p_{j}^{-1} \text { for all } i \in I_{\star} .
\end{aligned}
$$

That these sufficient conditions are also necessary, in general, is a consequence of the necessity of the hypotheses of Theorem 2.3.

Remark 7.1. Consider the case where each $X_{i}$ is a finite measure space. If $\left(p_{j}\right)_{j \in J}$ satisfies the hypothesis (2.15), and if $q_{j} \geq p_{j}$ for all $j \in J$, then $\Lambda\left(f_{j}\right)_{j \in J} \leq$ $C \prod_{j}\left\|f_{j}\right\|_{p_{j}} \leq C^{\prime} \prod_{j}\left\|f_{j}\right\|_{q_{j}}$ by Finner's theorem and Hölder's inequality. However, there are situations ${ }^{7}$ in which $\left(q_{j}\right)_{j \in J}$ satisfies (7.1) yet there exists no $\left(p_{j}\right)_{j \in J}$ satisfying (2.15) with $q_{j} \geq p_{j}$ for all $j \in J$.

To construct an example, begin with any situation where there is an extreme point $\left(q_{j}^{-1}\right)_{j \in J}$ of $K=\left\{\left(t_{j}\right)_{j \in J} \in[0,1]^{J}: 1=\sum_{j: i \in S_{j}} t_{j}\right.$ for all $\left.i \in I\right\}$, such that $q_{j}^{-1}<1$ for all $j$; for instance, the Loomis-Whitney example. Augment $I$ by adding a single new index $i^{\prime}$, choose one index $j^{\prime}$ already in $J$, and replace $S_{j^{\prime}}$ by $S_{j} \cup\left\{i^{\prime}\right\}$, while keeping $S_{j}$ unchanged for all $j \neq j^{\prime}$. Thus $\sum_{j: i^{\prime} \in S_{j}} q_{j}^{-1}=q_{j^{\prime}}^{-1}<1 ;\left(q_{j}\right)_{j \in S}$ satisfies (7.1). However no $\left(p_{j}\right)_{j \in J}$. For if $p_{j} \geq q_{j}^{-1}$ for all $j$ with strict inquality for some index $k$, choose some $i \in S_{k}$. Then $\sum_{j: i \in S_{j}} p_{j}^{-1}>\sum j: i \in S_{j} q_{j}^{-1}=1$, so that (7.1) fails for $\left(p_{j}\right)_{j \in S}$.

\footnotetext{
${ }^{7}$ The special case of Proposition 7.1 in which all $X_{i}$ are finite measure spaces is stated in [10], p. 1898 , but no proof is given.
} 
Proposition 7.1 can be proved by repeating Case 1 of the proofs of Theorems 2.1 and 2.3 , arguing by induction on $|I|$, and integrating with respect to the $m$-th coordinate in $\prod_{i \in I} X_{i}$ while all other coordinates are held constant. The basis case $m=1$ is Hölder's inequality. Indeed, this is the argument given in [10] for the special case when $I=I_{\star}$.

Alternatively, when $I_{0}$ is empty, ${ }^{8}$ Proposition 7.1 can be reduced to the case where each $X_{i}$ is $\mathbb{R}^{1}$ equipped with Lebesgue measure, by approximating general functions by finite linear combinations of characteristic functions of product sets, and then embedding any particular situation measure-theoretically into a (product of copies of) $\mathbb{R}^{1}$. The inequality (2.16) then follows from an application of Theorem 2.1.

\section{A final remark}

We have assumed throughout the discussion that all exponents satisfy $p_{j} \geq 1$. In Theorems 2.1, 2.2, and 2.3, the inequalities in question are false if some $p_{j}<1$. To see this, fix one index $j$. Take $f_{i}$ to be the characteristic function of a fixed ball centered at the origin for each $i \neq j$, take $f_{j}$ to be the characteristic function of a ball of measure $\delta$ centered at the origin, and let $\delta \rightarrow 0$. Then $\tilde{\Lambda}\left(f_{1}, \cdots, f_{m}\right)$ has order of magnitude $\delta$, while $\prod_{i}\left\|f_{i}\right\|_{L^{p_{i}}}$ has order of magnitude $\delta^{1 / p_{j}} \ll \delta$.

Valid inequalities can hold in Theorems 2.4 and 2.5 with some exponents strictly less than one, but they are always implied by stronger inequalities already contained in those theorems. More precisely, if the inequality holds for some $m$-tuple $\left(p_{1}, \cdots, p_{m}\right)$, then it also holds with each $p_{i}$ replaced by $\max \left(p_{i}, 1\right)$. In the case of Theorem 2.4 , that $p_{j}$ can be replaced by 1 if $p_{j}<1$ can be shown by considering the case when the support of $f_{i}$ is a single point, then exploiting linearity and symmetry.

\section{References}

[1] F. Barthe, On a reverse form of the Brascamp-Lieb inequality, Inv. Math. 134 (1998), 335-361.

[2] J. M. Bennett, A. Carbery and T. Tao, On the multilinear restriction and Kakeya conjectures, Acta Math. 196 (2006), no. 2, 261-302.

[3] J. M. Bennett, A. Carbery, M. Christ and T. Tao, The Brascamp-Lieb inequalities: finiteness, structure, and extremals, Geom. Funct. Anal. 17 (2008), no. 5, 1343-1415.

[4] H. J. Brascamp and E. H. Lieb, Best constants in Young's inequality, its converse, and its generalization to more than three functions, Advances in Math. 20 (1976), no. 2, 151-173.

[5] H. J. Brascamp, E. H. Lieb and J. M. Luttinger, A general rearrangement inequality for multiple integrals, J. Funct. Anal. 17 (1974), 227-237.

[6] A. P. Calderón, An inequality for integrals, Studia Math. 57 (1976), no. 3, 275-277.

[7] E. A. Carlen, E. H. Lieb and M. Loss, A sharp analog of Young's inequality on $S^{N}$ and related entropy inequalities, Jour. Geom. Anal. 14 (2004), 487-520.

[8] M. Christ, On certain elementary trilinear operators, Math. Res. Lett. 8 (2001), no. 1-2, 43-56.

[9] M. Christ, X. Li, T. Tao, and C. Thiele, On multilinear oscillatory integrals, nonsingular and singular, Duke Math. J. 130 (2005), no. 2, 321-351.

[10] H. Finner, A generalization of Hölder's inequality and some probability inequalities, Ann. Probab. 20 (1992), no. 4, 1893-1901.

[11] E. Friedgut, Hypergraphs, entropy, and inequalities, Amer. Math. Monthly 111 (2004), no. 9, 749-760.

[12] O. Hölder, Über einen Mittelwertssatz, Nachr. Acad. Wiss. Göttingen Math.-Phys. Kl., 1889, $38-47$.

\footnotetext{
${ }^{8}$ To treat the general case in this way would require a unification of Theorems 2.3 and 2.5 analogous to Proposition 7.1. We see no obstruction to such a result.
} 
[13] T. W. Hungerford, Algebra, Holt, Rinehart and Winston, Inc., New York-Montreal, Que.London, 1974.

[14] E. Lieb, Gaussian kernels have only Gaussian maximizers, Invent. Math. 102 (1990), no. 1, $179-208$

[15] L. H. Loomis and H. Whitney, An inequality related to the isoperimetric inequality, Bull. Amer. Math. Soc 55, (1949). 961-962.

[16] L. Maligranda, Why Hölder's inequality should be called Rogers' inequality, Math. Inequal. Appl. 1 (1998), no. 1, 69-83.

[17] L. J. Rogers, An extension of a certain theorem in inequalities, Messenger of Math. 17 (1888), 145-150.

School of Mathematics, University of Birmingham, Birmingham, B15 2TT, UK

E-mail address: J.Bennett@bham.ac.uk

School of Mathematics, University of Edinburgh, Edinburgh, EH9 3JZ, UK

E-mail address: A.Carbery@ed.ac.uk

Department of Mathematics, University of California, Berkeley, CA 94720-3840, USA

E-mail address: mchrist@math.berkeley.edu

Department of Mathematics, UCla, Los Angeles CA 90095-1555, USA

E-mail address: tao@math.ucla.edu 